\title{
Progressive Limb Weakness in A Young Man: A Case Report of POEMS Syndrome
}

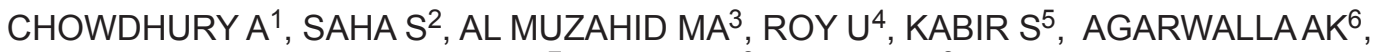 \\ REZA ${ }^{7}$, SARKER ${ }^{8}$,ISLAM MR ${ }^{8}$
}

\begin{abstract}
:
Polyneuropathy is an initial presentation and essential feature of POEMS ( polyneuropathy, organomegaly, endocrinopathy, M-protein, and skin changes) syndrome. Neuropathy is typically distal, symmetric and slowly progressive with demyelinating changes. After a gradual proximal spread, it usually results in severe muscle weakness and functional disabilities. In the present report, we describe a 40-year-old diabetic male presented with gradually progressive weakness of both lower limbs for 1 year followed by the involvement of both upper limbs for the last 3 months. On examination hyperpigmentation, lymphadenopathy, gynecomastia, anasarca, hepatomegaly, bilateral optic disc swelling, sensory-motor polyneuropathy was found. Laboratory findings showed IgG lambda monoclonal gammopathy, raised VEGF, sensory-motor demyelinating and axonal polyneuropathy. All findings were consistent with POEMS syndrome. The patient was treated with lenalidomide and dexamethasone cyclical therapy with some clinical improvement.
\end{abstract}

Keywords: POEMS syndrome, Progressive limb weakness, Polyneuropathy, VEGF

\section{Introduction:}

It is a rare, chronic, multisystemic, paraneoplastic disorder that occurs in the setting of plasma cell dyscrasia characterized by polyneuropathy, organomegaly, endocrinopathy, monoclonal plasma cell disorder and skin change ${ }^{1-4}$. The acronym, which was first coined by Bardwick in $1980^{1}$. However, this symptom complex is known with other names known as Osteosclerotic Myeloma, Takatsaki disease, Crow-Fukase syndrome. The real mechanism involved in the pathogenesis of POEMS syndrome is still unknown, but cytokines may play a major role ${ }^{9,10}$. Plasma and serum levels of VEGF are markedly increased in patients with POEMS and correlate with the activity of the disease ${ }^{6,7}$.
The POEMS acronym refers to several essential features of this syndrome. However, not all features within the acronym are required to make a diagnosis and additional important features are not included in the acronym ${ }^{4}$. For a strict diagnosis of POEMS syndrome, 2 mandate criteria of polyneuropathy and monoclonal plasma cell disorder must be present. Additional requirements for the POEMS diagnosis include at least 1 other major criterion of the sclerotic bone lesion, VEGF elevation or Castleman disease, and at least 1 minor criterion of organomegaly (hepatomegaly, splenomegaly and lymphadenopathy), endocrinopathy (hypogonadism, hyperestrogenemia, hypoparathyroidism etc), skin

1. Dr. Ashish Chowdhury, Phase B Resident, Dept. of Neurology, BSMMU, Dhaka

2. Dr. Sujan Saha, Phase B Resident, Dept. of Neurology, BSMMU, Dhaka

3. Dr. Md. Abdullah Al Muzahid, Phase B Resident, Dept. of Neurology, BSMMU, Dhaka

4. Dr. Uttam Roy, Phase B Resident, Dept. of Neurology, BSMMU, Dhaka

5. Dr. Md. Suman Kabir, Phase B Resident, Dept. of Neurology, BSMMU, Dhaka

6. Dr. Ajay Kumar Agarwalla, Assistant Register, Dept. of Neurology, SSMCH, Dhaka

7. Dr. Samim Reza, Phase B Resident, Dept. of Haematology, BSMMU, Dhaka

8. Dr. Imran Sarker, Assistant Professor(CC), Dept. of Clinical Neurology, NINS\&H, Dhaka

9. Prof. Dr. Md. Rafiqul Islam, Professor and Chairman, Dept. of Neurology, BSMMU, Dhaka 
change, edema, polycythemia/thrombocytosis or papilledema ${ }^{2,4}$.

The diagnosis of POEMS syndrome is made based on a composite of clinical and laboratory features. It is a rare disorder with a reported prevalence of approximately 0.3 per $100,000^{3}$. The peak incidence of the POEMS syndrome is in the fifth and sixth decades of life, unlike multiple myeloma (MM), which has a peak incidence in the seventh and eighth decades ${ }^{4}$. A male preponderance also observed with POEMS ${ }^{4}$. A good history and physical examination followed by appropriate testing, most notably radiographic assessment of bones, measurement of vascular endothelial growth factor (VEGF), careful analysis of a bone marrow biopsy can differentiate this syndrome from other conditions like CIDP, monoclonal gammopathy of undetermined significance (MGUS), neuropathy, immunoglobulin light chain amyloid neuropathy. There is a Castle- man variant of POEMS syndrome that does not have a clonal plasma cell proliferative disorder underlying but has many of the other paraneoplastic features. ${ }^{12-14}$

Peripheral polyneuropathy in POEMS is usually symmetrical ascending sensorimotor polyneuropathy. The polyneuropathy usually begins with sensory changes in the lower extremities, then progressing to the motor deficit. As demonstrated in this case report, the course of polyneuropathy is usually a steady progression, at times more rapidly, until appropriate treatment is given to the underlying plasmacytoma ${ }^{4}$.

\section{Case Report:}

A 40-year-old man, college teacher, nonsmoker, non-alcoholic, normotensive but diabetic healing from Cumilla was admitted into the department of neurology, BSMMU, Dhaka, Bangladesh. He was presented with the complaints of weakness of both lower limbs for the last 1 year and swelling of both legs for the last 3 months. According to the patient, he was reasonably well about 1 year back, then he developed weakness in both lower limb, which was insidious onset, gradually progressive, symmetrical and involved distal parts. Initially, the symptom was mild, but for the last 3 months weakness became so severe that the patient could not walk or even stand without help from others and it gradually involved the upper limbs also. on a query, he also complained about tingling and numbness of all four limbs for the same duration. He also noticed swelling of both feet for the last 3 months and some painless lump on his left side of the neck for around 9 months. He denied any history of fever, anorexia, cough, breathlessness, weight loss, night sweat, body aches, joint pain, skin rash, jaundice, scanty micturition, headache, visual disturbance or dizziness or difficulty in standing from sitting. He was not a strict vegan and his bowel and bladder habit was normal. $\mathrm{He}$ is diabetic for the last 10 years, initially on OHA but now on insulin. For his illness, he visited several physicians in Bangladesh and abroad. He underwent several biochemical, hematological and neurological investigations and treated accordingly but no clinical improvement occurred. No significant drug history. But for his illness, he took Pregabalin, Multivitamin and Vit$\mathrm{B} 1,6,12$. He is blessed with 3 children. He had 8 siblings. His parents were alive. All were in good health with no significant symptoms.

At the time of presentation to us, he was alert with a body built below average. Vitals were within normal limits with no anemia, jaundice, clubbing, thyromegaly, bony tenderness. But the patient had some hyperpigmentation lesions in different parts of body. The patient had firm, discrete, multiple, non-tender, left anterior cervical lymphadenopathy. Gynecomastia and bi-pedal pitting edema were also present. Regarding neurological examinations higher cerebral function including speech was normal. All cranial nerves were intact except fundoscopy revealed bilateral optic disc swelling. Motor examination revealed bilateral symmetrical wasting of both lower limbs, no fasciculation but bilateral foot drop was present. Muscle tone was reduced in all four limbs. Muscle power was MRC grade 4 in upper limbs both proximally and distally. In the lower limbs muscle power was MRC grade 3 proximally and MRC grade 2 distally. All deep tendon reflexes were diminished, plantar response was bilateral flexor, gait could not be evaluated due to weakness. Cerebellar function was intact. Hyperesthesia in gloves and stocking pattern was present in both upper and lower limbs. On 
Abdominal examination, nontender firm hepatomegaly and ascites was present. No other organomegaly found and all other systemic examinations revealed no significant abnormality.

Regarding laboratory investigations, $\mathrm{CBC}$ showed thrombocytosis (platelet $690,000 \mathrm{cu} / \mathrm{mm}$ ), hypoalbuminemia and hyperglobulinaemia, raised alkaline phosphatase level, $\mathrm{HbA}_{1} \mathrm{C}-7.9 \%$. Ultrasound of the abdomen showed hepatomegaly, ascites, mild bilateral pleural effusion. Thyroid function test, renal function test, bilirubin, other liver function test, electrolytes, serum vitamin $\mathrm{B}_{12}$ level, tuberculin test, ANA, Viral markers were found within normal limit. Nerve conduction study of all four limbs showed severe sensory-motor demyelinating and axonal polyneuropathy of both upper and lower limbs ( $L L>U L$ ) without any conduction block. The biopsy of the left cervical lymphnode showed reactive hyperplasia. Serum protein immunotyping showed IgG lambda monoclonal gammopathy, VEGF level raised (477 $\mathrm{pg} / \mathrm{ml}$; reference range, $31-86 \mathrm{pg} / \mathrm{ml}$ ). Bone marrow aspiration cytology-normocellular marrow with plasmocytosis ( plasma cell $<5 \%$ ) and trilineage hematopoiesis, Bone marrow trephine biopsy- mild increase in a clonal plasma cell. Xray pelvis shows some osteosclerotic lesions. Whole-body PET-CT revealed multiple enlarge metabolically active cervical, supraclavicular, axillary lymphnode and destructive lesion in dorsal, lumbar, left ischium, left lamina of $\mathrm{C}_{2}$ vertebrae.

Pharmacologically he was treated lenalidomide and dexamethasone as 28 days cycle

lenalidomide $10 \mathrm{mg}$ day $1-21$ and dexamethasone $40 \mathrm{mg}$ per day for 4 days in every wk for the first cycle ). His tingling and numbness were improved, edema subsided but no significant improvement of motor function

\section{Discussion:}

Neurologists are frequently at the forefront of diagnosing POEMS syndrome. Diagnosis of POEMS is often delayed due to its rarity, lack of all the typical features and physicians' unfamiliarity with the entity.

POEMS syndrome differs from other paraproteinaemic and inflammatory neuropathies by its multi-organ involvement thought to be caused by elevated pro-inflammatory and angiogenic cytokines. As already stated, multi-organ features extend beyond those included in its acronym, and not all features included in the acronym are required for diagnosis ${ }^{2-6,21}$.

Polyneuropathy is an initial presentation and essential feature of POEMS syndrome ${ }^{2-4}$. Initial presentation of our patient also consistent with features of polyneuropathy. Patients typically present with a subacute, distal, symmetrical, sensorimotor neuropathy, frequently painful, with allodynia and hyperpathia ${ }^{3,22}$. The lower limbs are affected earlier, and more severely than the upper limbs $3,23,24$. Sensory symptoms usually precede motor symptoms ${ }^{23}$. s. Many patients quickly become wheelchair- or bed-bound due to weakness or pain. The clinical examination may reveal distal wasting, weakness and sensory loss affecting both large and small fibre sensory modalities $^{3}$. All these features were similar to our patient except sensory symptoms were started simultaneously with motor symptoms.

Electrodiagnostic studies demonstrate a lengthdependent sensorimotor neuropathy, typically demyelinating, but with axonal degeneration. Conduction block is tended not usually present. In motor studies, reduction in motor conduction velocity (MCV) is an early sign, however, patients often already have a significant axonal loss at presentation ${ }^{22-24}$. Sensory studies show a reduction of, or often absent, sensory nerve action potentials ${ }^{3,22-24}$. Conduction block is much more common in CIDP than POEMS syndrome and the discrepancy in severity between upper and lowers limb axonal loss is more pronounced in POEMS syndrome ${ }^{3,25}$. Findings were similar to our patient showing absent SNAP in sural nerve and reduction of SNAP in the median and ulnar nerve. Prolonged latency and amplitude in median and ulnar nerve, no CMAP in the lower limb. No conduction block.

The constellation of neuropathy and any of the following should elicit an in-depth search for POEMS syndrome: monoclonal protein (especially lambda light chain), thrombocytosis, anasarca, or papilledema. Any patient who carries a diagnosis 
of chronic inflammatory demyelinating polyneuropathy (CIDP) that is not responding to standard CIDP therapy should be considered as possibly having POEMS syndrome, and additional testing should be done to rule in or rule out the diagnosis of POEMS syndrome ${ }^{19,20}$. At the beginning of presentation, CIDP is also our concern but we examine and investigate to reach an appropriate diagnosis. The M protein is usually immunoglobulin (Ig) $\mathrm{G}$ or $\lg A$ and almost always of the lambda type ${ }^{11-13}$. Laboratory findings are notable for the absence of cytopenias. Nearly half of the patients have thrombocytosis or erythrocytosis ${ }^{11-13}$. Bone marrow usually contains less than $5 \%$ plasma cells, and, when clonal cells are found, they are almost always monoclonal lambda. Little is known about the plasma cells in POEMS syndrome except that more than $95 \%$ of the time they are lambda light chain restricted with restricted immunoglobulin light chain 15,26 . The bone marrow biopsy reveals megakaryocyte hyperplasia and megakaryocyte clustering in 54\% and $93 \%$ of cases, respectively ${ }^{11}$. We have similar findings in our patient, IgG lambda monoclonal gammopathy, plasma cell $5 \%$, thrombocytosis, increased megakaryopoiesis.

Osteosclerotic lesions occur in approximately 95\% of patients and can be confused with benign bone islands, aneurysmal bone cysts, nonossifying fibromas, and fibrous dysplasia ${ }^{19,27}$. Some lesions are densely sclerotic, whereas others are lytic with a sclerotic rim, whereas still others have a mixed soap-bubble appearance. FDG uptake occurs in those lesions that have a lytic component ${ }^{28}$. Lesions are commonly found in the pelvis, thoracic and lumbar vertebrae, and ribs, and also occur in the scapula, clavicle, sternum, skull and long bones normal. In our patient X-ray pelvis shows some osteosclerotic lesion and PET -CT of whole body revealed expansile/permeative bony destruction of thoracic, lumbar vertebrae, left ischium. In a retrospective cohort of 29 patients diagnosed with POEMS syndrome in India between 1983 and 2009, all had organomegaly, including hepatomegaly (28/29), splenomegaly (21/29) and lymphadenopathy (7/29). In our case, we found both hepatomegaly and lymphadenopathy but no splenomegaly found.

In a retrospective study of 64 patients with POEMS syndrome, $84 \%$ had endocrinopathy, $54 \%$ of whom had multiple endocrinopathies. Hypogonadism was commonest, affecting $79 \%$ of men. Other endocrine abnormalities described include thyroid dysfunction, abnormal calcium metabolism, glucose intolerance, diabetes, hyperprolactinemia, gynecomastia, and less commonly adrenal insufficiency 29,30 . In our case, we found diabetes mellitus, gynecomastia but thyroid function was normal and other hormonal evaluation was not done.

Skin changes are reported in as many as $90-100 \%$ of patients ${ }^{7,10}$. Hyperpigmentation and haemangiomas, thickening, hypertrichosis, acquired facial lipoatrophy, and infiltrated livedo reticularis with necrosis, acrocyanosis, flushing, rubor, hyperemia, and Raynaud's phenomenon may be the presentation. In our patient, we found multiple hyperpigmented lesions in different parts of body.

Helpful cut-offs for plasma and serum VEGF levels to diagnosis POEMS syndrome are $200 \mathrm{pg} / \mathrm{mL}$ (specificity 95\%; sensitivity 68\%) ${ }^{9}$ and 1920 pg/ $\mathrm{mL}$ (specificity $98 \%$; sensitivity $73 \%$ ), ${ }^{9}$ respectively. The best cut-off of $\mathrm{N}$-terminal propeptide of type I collagen to diagnosis POEMS syndrome $70 \mathrm{ng} /$ $\mathrm{mL}$ with a specificity of $91.5 \%$ and a sensitivity of $80 \%{ }^{20}$. In our patient VEGF level was raised (477 $\mathrm{pg} / \mathrm{ml}$; reference range, $31-86 \mathrm{pg} / \mathrm{ml}$ ).

Evidence for treatment in POEMS syndrome is largely limited to retrospective cohort studies, with only one randomized controlled trial (RCT) to date. IVIg or steroid monotherapy, commonly used in other inflammatory neuropathies, does not produce a lasting benefit. The current suggested treatment algorithm recommends localized radiotherapy for patients with localized disease, defined as up to 3 discrete bone lesions and no evidence of clonal plasma cells on iliac crest biopsy, or systemic treatment in patients with diffuse disease, defined as $>3$ bone lesions or clonal plasma cells on iliac crest biopsy ${ }^{31}$. Systemic treatment options include ASCT or chemotherapy. Supportive treatment for neurological disability and systemic symptoms 
should also be considered ${ }^{31,32}$. Lenalidomide is structurally similar to thalidomide but less neurotoxic. Two recent prospective studies, some small retrospective studies and a pooled analysis study have all shown it to be effective in POEMS syndrome, as either first- or second-line therapy 31,32 . Our patient having clonal plasma cell and multiple bony lesions with start lenalidomide and dexamethasone cyclical therapy .

\section{Conclusion:}

Though POEMS syndrome is a very rare, but treatable cause of neuropathy. Further work is required to establish its exact underlying pathophysiology. Current treatment approaches afford a good prognosis. Moving forward, randomized-controlled studies, though difficult given the rarity of POEMS syndrome, and the development of prognostic tools will be important in establishing individualized approaches to patient care. Most importantly if any suspected CIDP patient not improving with usual treatment, we should must exclude POEMS syndrome.

Conflict of interests: The authors declare that they have no conflict of interest

\section{References:}

1. Bardwick PA, Zvaifler NJ, Gill GN, Newman D, Greenway GD, Resnick DL. Plasma cell dyscrasia with polyneuropathy, organomegaly, endocrinopathy, M protein, and skin changes: the POEMS syndrome. Report on two cases and a review of the literature. Medicine. 1980;59(4):311-322.

2. Dispenzieri A. POEMS syndrome: update on diagnosis, risk-stratification, and management. Am J Hematol 2015;90(10):951-62.

3. S. Nasu, S. Misawa, Y. Sekiguchi, et al., Different neurological and physiological profiles in POEMS syndrome and chronic inflammatory demyelinating polyneuropathy, J. Neurol. Neurosurg. Psychiatry 83 (5) (2012) 476-479.

4. DispenzieriA (2017) POEMS syndrome: 2017 Update on diagnosis, risk stratification, and management. Am J Hematol 92:814-829
5. DispenzieriA et al (2003) POEMS syndrome: Definitions and long-term outcome. Blood 101:2496-2506

6. Nasu S et al (2012) Different neurological and physiological profiles in POEMS syndrome and chronic inflammatory demyelinating polyneuropathy. J Neurol Neurosurg Psychiatry 83:476-479

7. Watanabe $\mathrm{O}$, Maruyama I, Arimura $\mathrm{K}$, et al. Overproduction of vascular endothe- lial growth factor/vascular permeability factor is causative in Crow-Fukase (POEMS) syndrome. Muscle Nerve 1998;21(11):13907.

8. Scarlato M, Previtali SC, Carpo M, et al. Polyneuropathy in POEMS syndrome: role of angiogenic factors in the pathogenesis. Brain 2005;128(Pt 8):1911-20.

9. Nobile-Orazio E, Terenghi F, Giannotta C, et al. Serum VEGF levels in POEMS syndrome and in immune-mediated neuropathies. Neurology 2009;72(11): 1024-6.

10. Watanabe O, Arimura K, Kitajima I, et al. Greatly raised vascular endothelial growth factor (VEGF) in POEMS syndrome [letter]. Lancet 1996;347(9002):702

11. Takatsuki K, Sanada I. Plasma cell dyscrasia with polyneuropathy and endo- crine disorder: clinical and laboratory features of 109 reported cases. Jpn J Clin Oncol 1983;13(3):543-55.

12. Alberti MA, Martinez-Yelamos $S$, Fernandez $A$, et al. 18F-FDG PET/CT in the evaluation of POEMS syndrome. Eur $J$ Radiol 2010;76(2):180-2.

13. Glazebrook K, Guerra Bonilla FL, Johnson A, et al. Computed tomography assessment of bone lesions in patients with POEMS syndrome. Eur Radiol 2015;25(2):497-504.

14. Shi X, Hu S, Luo X, et al. CT characteristics in 24 patients with POEMS syn- drome. Acta Radiol 2016;57(1):51-7

15. Soubrier $M$, Labauge $P$, Jouanel $P$, et al. Restricted use of Vlambda genes in POEMS 
syndrome. Haematologica 2004; 89(4): ECR02

16. Briani C, Fabrizi GM, Ruggero $S$, et al. Vascular endothelial growth factor helps differentiate neuropathies in rare plasma cell dyscrasias. Muscle Nerve 2010; 43(2): 164-7.

17. D'Souza A, Hayman SR, Buadi F, et al. The utility of plasma vascular endothelial growth factor levels in the diagnosis and follow-up of patients with POEMS syn- drome. Blood 2011;118(17):4663-5.

18. Dao LN, Hanson CA, Dispenzieri A, et al. Bone marrow histopathology in POEMS syndrome: a distinctive combination of plasma cell, lymphoid and myeloid findings in 87 patients. Blood 2011;117(24):6438-44.

19. Dispenzieri, A., Kourelis, T. and Buadi, F., 2018. POEMS syndrome: diagnosis and investigative work-up. Hematology/Oncology Clinics, 32(1), pp.119-139.

20. Dispenzieri, A., 2019. POEMS Syndrome: 2019 Update on diagnosis, risk stratification, and management. American journal of hematology, 94(7), pp.812-827.

21. Dispenzieri A. Castleman disease. Cancer Treat Res 2008;142:293-330

22. Koike $\mathrm{H}$ et al (2008) Neuropathic pain correlates with myelinated fibre loss and cytokine profile in POEMS syndrome. $J$ Neurol Neurosurg Psychiatry 79:1171-1179

23. Kulkarni GB et al (2011) Clinicopathological profile of poly-neuropathy, organomegaly, endocrinopathy, $M$ protein and skin changes (POEMS) syndrome. J Clin Neurosci 18:356360
24. Liu M et al (2015) Motor nerve conduction study and muscle strength in newly diagnosed poems syndrome. Muscle Nerve 51:19-23

25. Sung JY, Kuwabara S, Ogawara K, Kanai K, Hattori T (2002) Patterns of nerve conduction abnormalities in POEMS syndrome. Muscle Nerve 26:189-193

26. Nakaseko C, Abe D, Takeuchi M, et al. Restricted oligo-clonal usage of mono- clonal immunoglobulin $\{l a m b d a\}$ light chain germline in POEMS syndrome. ASH Annual Meeting Abstracts 2007;110(11):2483

27. Nakanishi T, Sobue I, Toyokura Y, et al. The Crow-Fukase syndrome: a study of 102 cases in Japan. Neurology 1984;34(6):712-20

28. Pan Q, Li J, Li F, et al. Characterizing POEMS syndrome with $18 \mathrm{~F}$-fludeoxyglu- cose positron emission tomography/computed tomography. J Nucl Med 2015; 56(9):1334-7.

29. Mangalik A, Veliath AJ. Osteosclerotic myeloma and peripheral neuropathy. A case report. Cancer 1971;28(4):1040-5.

30. Evison G, Evans KT. Sclerotic bone deposits in multiple myeloma [letter]. $\mathrm{Br} \mathrm{J}$ Radiol 1983;56(662):145.

31. Misawa S et al (2016) Safety and efficacy of thalidomide in patients with POEMS syndrome: a multicentre, randomised, doubleblind, placebo-controlled trial. Lancet Neurol 15:1129-1137

32. Kuwabara S, Dispenzieri A, Arimura K, Misawa S, Nakaseko C (2012) Treatment for POEMS (polyneuropathy, organomeg-aly, endocrinopathy, M-protein, and skin changes) syndrome. Cochrane Database Syst Rev. https://doi.org/10.1002/14651858. CD006828.pub3 